\title{
OSTEOPONTIN SIRNA DOES NOT CONFER RESISTANCE TO TOXIC EFFECTS OF PARTHENOLIDE IN JURKAT CELLS
}

\author{
S. Mehri', S. Mohammadi', M. Nikbakht ${ }^{2}$, M. Sahmani ${ }^{1}$, M. Zahedpanah ${ }^{1, *}$ \\ ${ }^{1}$ Qazvin University of Medical Sciences, Qazvin, 34197-59811, Iran \\ ${ }^{2}$ Hematology, Oncology and Stem Cell Transplantation Research Center, Tehran University \\ of Medical Sciences, Tehran 14155-6559, Iran
}

\begin{abstract}
Background: Osteopontin (OPN) plays a critical role in cell proliferation and drug resistance in cancer treatment and hematological malignancies. In T cell acute lymphoblastic leukemia, most initial therapies can induce remission while some patients then relapse and do not respond well to chemotherapy. The sesquiterpene lactone parthenolide (PTL) can induce apoptosis in a variety of cancer cell lines via inhibition of pro-inflammatory transcription factor nuclear factor kappa $B$ and has anti-tumor activity in acute lymphoblastic leukemia treatment. Aim: To study the role of OPN in conferring in vitro resistance to PTL in Jurkat cells. Methods: Jurkat cells were cultured with 8-20 $\mu \mathrm{m}$ PTL for $48 \mathrm{~h}$. Transfection with OPN siRNA was provided. Apoptosis assays were performed with Annexin V-Alexa Fluor-488/PI. Quantitative real-time polymerase chain reaction was used to measure $O P N$ gene

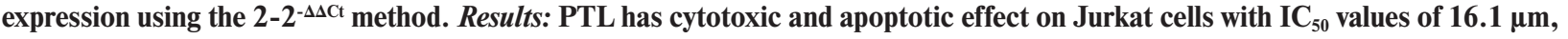
and growth inhibition effect of PTL does not differ significantly in combination with OPN-siRNA. OPN gene expression is not affected by PTL. Conclusions: Parthenolide induces apoptosis in Jurkat cells, but inhibition of osteopontin gene expression with siRNA does not reduce apoptotic effect of parthenolide.
\end{abstract}

Key Words: Jurkat cells, osteopontin, parthenolide.

DOI: 10.32471/exp-oncology.2312-8852.vol-42-no-3.15180

Osteopontin (OPN), also known as secreted phosphoprotein 1 is a $34 \mathrm{kD}$ multidomain secreted glycoprotein that functions as a soluble cytokine and an adhesive component of the extracellular matrix [1, 2]. It is a member of small integrin-binding ligand $\mathrm{N}$-linked glycoproteins (siblings), a family of five integrin binding glycophosphoproteins [3]. OPN interacts mainly with various $\alpha_{v}$ (particularly $\alpha_{v} \beta_{1}, \alpha_{v} \beta_{3}, \alpha_{v} \beta_{5}$ ) integrins and with the CD44 splice variants. Due to these properties, OPN induces the activation of signal transduction pathways leading to cell proliferation, adhesion, invasion and migration. Hence, its role in cancer is important, as it is involved in angiogenesis, and metastasis [4-7]. OPN, also called early T cell activation gene 1 (Eta-1), is expressed after activation of T cells, macrophages and in multiple tissue types. OPN is cleaved by some proteases such as thrombin, cleaved product participating in various cellular functions. Jurkat cells originating from $T$ cell acute lymphoblastic leukemia (T-ALL) have enhanced cell adhesion to thrombincleaved OPN compared with full-length OPN $[8,9]$.

Given that the human $O P N$ gene is encoded by a single-copy gene on chromosome 4q21-q25 and has an alternative translation start site, there are two major variants of OPN, intracellular and extracellular [1]. It is also subjected to alternative splicing as well as post-translational modifications such as phosphorylation, glycosylation and proteolytic cleavage.

\footnotetext{
Submitted: September 30, 2019.

*Correspondence: E-mail: zhdpnh@yahoo.com Abbreviation used: ALL - acute lymphocytic leukemia; DMSO dimethyl sulfoxide; OPN - osteopontin; PI - propidium iodide; PCR - polymerase chain reaction; PTL - parthenolide; siRNA short interfering RNA; T-ALL - T-cell acute lymphoblastic leukemia.
}

Parthenolide (PTL)-containing herbs are highly bioactive and have been traditionally used for treatment of inflammations. The anti-inflammatory activities of these herbs are partially due to their anti-NFkB effects [10]. Currently PTL is used extensively in cancer treatment [11-14]. It can induce oxidativestress-mediated apoptosis in T cells at high doses (up to $10 \mu \mathrm{m}$ ) [15].

T-ALL represents approximately $12 \%$ to $15 \%$ of all newly diagnosed acute lymphocytic leukemia (ALL) cases in pediatric patients and about $25 \%$ of adult ALL. This aggressive malignancy is associated with a significant risk of disease relapse and does not respond well to chemotherapy [16-18].

Herein, we attempted to study the role of OPN in conferring in vitro resistance to PTL in Jurkat cells and to examine the effect of knockdown of OPN gene expression via OPN-siRNA on PTL-treated Jurkat cell survival.

\section{MATERIALS AND METHODS}

Reagents. Parthenolide was purchased from Sigma-Aldrich (USA) and dissolved in dimethyl sulfoxide (DMSO) as a $50 \mathrm{mM}$ stock solution, stored at $-20^{\circ} \mathrm{C}$, and diluted in DMSO before use. The Annexin V-FITC/ PI kit was purchased from BD Biosciences (USA). Tripure Isolation Reagent was purchased from Roche Applied Science (Germany). The cDNA synthesis kit and SYBR ${ }^{\circledR}$ Premix Ex Taq ${ }^{\mathrm{TM}}$ were purchased from Takara Biotechnology Co. (Otsu, Japan).

Cell culture. The human leukemic Jurkat cell line was obtained from the Iranian Biological Resource Center. RPMI 1640 supplemented with $10 \%$ fetal bovine serum medium (Invitrogen, USA) was used for culturing. The medium was supplemented with

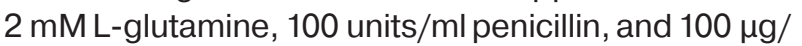

